\title{
Genetix
}

Take the lead

\section{Rapid isolation of antigen-specific clones from hybridoma fusions}

\author{
The Genetix ClonePix FL is a powerful way to find and collect antigen-specific clones from hybridoma \\ fusions in a rapid one-step process - shortening the process timeline and allowing parallel \\ interrogation of multiple antigens in the monoclonal antibody discovery process. ClonePix FL \\ automatically screens the entire hybridoma fusion in situ and isolates only antigen-specific clones with \\ the desired immunoglobulin isotype. If the fusion has many antigen-positive clones, then ClonePix FL \\ can selectively collect only the best secretors.
}

Screening a hybridoma fusion and then subcloning candidate hybrids are time-consuming elements of the monoclonal antibody discovery process. The Genetix ClonePix FL provides a powerful way to find and collect antigen-specific clones in a rapid one-step process. The technology works by automatically screening the entire fusion in situ and isolating only antigen-specific clones with the desired immunoglobulin isotype. If the fusion has many antigen-positive clones, then ClonePix FL can selectively collect only the best secretors. The gentle nature of the technology ensures high viability of the selected clones (90-100\%). By eliminating traditional methods and minimizing the labor required, ClonePix FL shortens the process timeline. Furthermore, it permits parallel interrogation of multiple antigens.

Plating hybridomas into methylcellulose-based semisolid medium was first described 25 years ago ${ }^{1}$. Building on this method, Genetix has developed a technology that traps, detects and measures the relative secretion of monoclonal antibody from the hybridomas (or transfected cells such as myelomas). As the clonal colonies grow suspended in semisolid medium, the secreted antibody is immobilized in the vicinity of the colony by isotype-specific Complex Initiation Factor (CIF), and specific clones are then detected by probing with a fluorescent antigen (Fig. 1). During imaging, up to five fluorescence wavelengths can be multiplexed with brightfield images, thus allowing the use of multiple probes in a single picking run or the measurement of other parameters such as cell viability (using the Genetix LiveDetect reagent).

\section{Selection of antigen-specific clones}

After fusion, allow the hybridomas to recover for $24 \mathrm{~h}$ before plating them out. First, prepare the semisolid medium with all required

\section{Christopher J Mann}

Genetix Ltd., Queensway, New Milton, Hampshire BH25 5NN, UK. Correspondence should be addressed to C.J.M. (chris.mann@genetix.com).

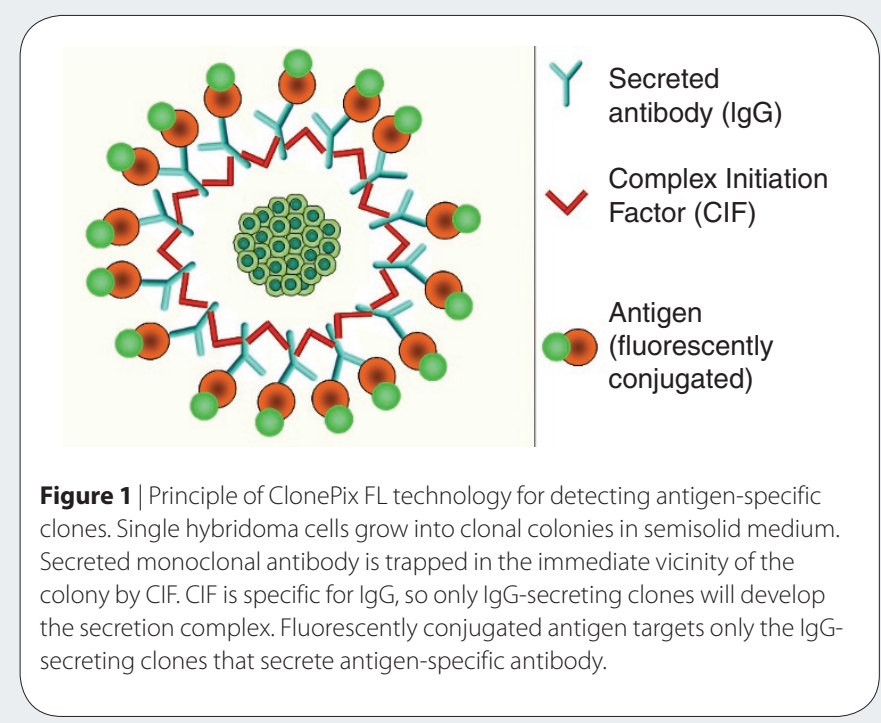

components. For convenience, CloneMedia semisolid medium containing serum and cloning supplements is available in 100-ml quantities, ideal for working with one fusion. Alternatively, semisolid medium can be prepared by adding a $2 \times$ medium concentrate of your choice to CloneMatrix. Before adding cells, supplement the medium with any additional components (such as antibiotics, hypoxanthine, aminopterin and thymidine (HAT) selection agents), and then add $100 \mathrm{U} / \mathrm{ml}$ of $\mathrm{CIF}$ and your fluorescently conjugated antigen of interest. The concentration of antigen required is dependent on its properties (molecular weight and dye-to-protein ratio). As a general guideline, Genetix recommends using $0.1-10 \mu \mathrm{g} / \mathrm{ml}$ of antigen, but this should be determined empirically. After gentle but thorough mixing, the hybridomas can be added. Distribute the cells evenly by rotating the bottle, and then plate the contents. Genetix PetriWell- 6 and PetriWell- 1 plates are designed specifically for this application to minimize the autofluorescence and flaring that occurs when regular culture plates 


\section{a}
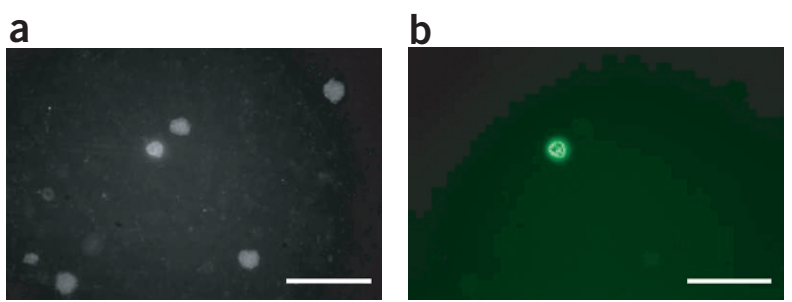

Figure $\mathbf{2}$ | ClonePix FL selectively detects hybrids secreting antigen-specific $\operatorname{lgG}$. (a,b) Cells were plated and treated as described in Figure $\mathbf{1}$ using a FITC-conjugated 60-kDa bacterial antigen. Brightfield image (a). Fluorescence image (excitation $470 \pm 20 \mathrm{~nm}$; emission $535 \pm 25 \mathrm{~nm}$; b). Only clones secreting antigen-specific lgG display local fluorescence. Specificity was confirmed by antigen-specific enzyme-linked immunosorbent assay (data not shown). Scale bars, $1 \mathrm{~mm}$.

are imaged. The plates also contain reservoirs to place a small volume of sterile water to minimize evaporation during incubation.

After 7-8 days of incubation, a plated fusion should generate at least 3,000 discrete, HAT-selected hybrid colonies of 100-500 cells each. Using ClonePix FL, the fluorescing clones (in other words, the clones that are secreting antigen-specific immunoglobulin gamma (IgG); see Fig. 2) are automatically targeted for collection and then picked into separate wells of a 96-well destination plate containing the medium of choice for outgrowth (for example, XP Media). The software excludes any clones that are too close to each other (by brightfield imaging), thereby preventing nonclonality. The user has the option of reviewing and adjusting the parameters of the selected clones before picking them.

If there is concern about the stability of the fluorescent antigen during the growth period or if accumulation of the fluorescent antigen around unfused B cells is an issue, one or both of the probes can be added after colony growth by using an atomizer applicator $24 \mathrm{~h}$ before imaging and picking the colonies.

\section{Selection of highest-secreting antigen-specific clones}

The above method is designed for finding rare antigen-positive hybrids. If a fusion is expected to have many antigen-positive clones, ClonePix FL can selectively collect only the best secretors. In a multifluorescence variation of the above method, CIF can be replaced by CloneDetect detection agent, which is a superior indicator of the clone production rate. The CloneDetect agent is a complex-initiation probe specific for mouse $\operatorname{lgG}$ and is available conjugated with a variety of fluorophores. In this application, the fluorescent antigen will detect the antigenpositive clones, and the CloneDetect will quantify the amount of $\lg G$ secreted.

\section{Selection of highest secreting lgG-specific clones}

If it is not possible to add a fluorescently conjugated antigen-for example, if the antigen has poor solubility - the assay can still be run using just CloneDetect reagent. Under these conditions, only clones
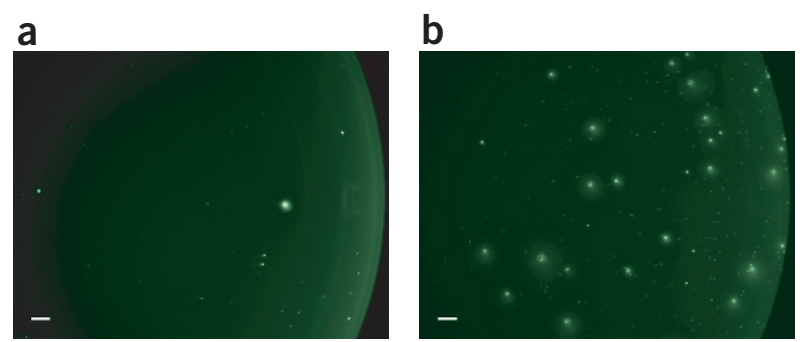

Figure 3 Recovering a failing hybridoma cell line. (a) A poorly secreting cell line was screened on ClonePix FL, which showed broad heterogeneity of secretion between subclones at day 5. (b) The highest-fluorescing clones were picked by ClonePix FL, and subsequent replating showed good subclone fluorescence. Scale bars, $1 \mathrm{~mm}$.

producing $\lg \mathrm{G}$ isotype monoclonal antibodies will be detected and collected, thus eliminating IgM-secretors or nonsecretors.

\section{Subcloning}

Subcloning is not required normally, but can lead to isolation of a considerably higher-producing cell line. Using ClonePix FL, the entire subcloning process can be completed in one week by transferring the contents of a picked well into a small amount of semisolid medium (2-4 $\mathrm{ml}$ ) containing CloneDetect reagent and replating. After 5-7 days of growth, the subclones can be re-imaged and the highest secretors collected. The collection of a clonal colony rather than a single cell ensures rapid expansion. This simple procedure is also valuable for resurrecting existing hybridoma cultures that are failing to secrete (Fig. 3).

\section{Conclusions}

Using ClonePix FL technology removes the need for serial dilution and overcomes the limitations of cell sorters by directly detecting and measuring antibody secretion in situ. It allows monoclonal populations of antigen-specific, high secreting, IgG isotype hybrids to be established in a much shorter time frame, removing many of the bottlenecks associated with traditional methods. We have validated the technology for a broad range of antigens, from a $160-k D a$ multimeric protein to a 2.6-kDa phosphopeptide. The low labor requirement means that more hybridoma fusions can be interrogated in parallel, increasing cell line throughput. We estimate that two scientists using a ClonePix FL system should be able to process up to 100 fusions per year.

The complete list of products is available in the ClonePix FL reagents and consumables brochure. Additional information is available on our company website (http://www.genetix.com/clonepix).

1. Davis, J.M., Pennington, J.E., Kubler, A.M. \& Conscience, J.F. A simple, singlestep technique for selecting and cloning hybridomas for the production of monoclonal antibodies. J. Immunol. Methods 50, 161-171 (1982).

This article was submitted to Nature Methods by a commercial organization and has not been peer reviewed. Nature Methods takes no responsibility for the accuracy or otherwise of the information provided. 